\title{
Tabuthema: Gestörte Sexualität bei Diabetikern
}

\author{
Diabetiker leiden sehr viel häufiger unter einem gestörten Liebesleben als Stoffwechselgesunde. \\ Eine unabdingbare Voraussetzung für den Erfolg einer Therapie sind bei diesen oft tabuisierten \\ Problemen die einfühlsame Kommunikation unter Einbeziehung des Partners.
}

_ Es ist davon auszugehen, dass bei jedem zweiten Diabetiker eine Störung der Sexualfunktion vorliegt - Männer und Frauen sind gleichermaßen betroffen, so Dr. Astrid Tombek, Bad Mergentheim.

\section{Probleme werden oft totgeschwiegen} Die häufigste Sexualstörung bei Diabetikerinnen sind Lubrikationsstörungen und Dyspareunie. Auch eine gesteigerte Anfälligkeit für urogenitale Infektionen, die durch SGLT2-Hemmer verstärkt wird, kann bei Frauen einen störenden Einfluss auf die Sexualtät haben. Die Probleme werden oft totgeschwiegen.

Bei allgemeiner sexueller Unlust müssen bei Frauen daneben vielfältige Ursachen diskutiert werden. „Es liegt nicht immer nur am Diabetes“, so Tombek. Auch hormonelle Störungen, Sucht- und andere psychische Erkrankungen sowie Medikamente können die Ursache sein. Dazu kommen soziokulturelle Faktoren und vor allem Beziehungsfaktoren, also Stress und Partnerschaftsprobleme.

\section{ED-Risiko vierfach erhöht}

Bei Männern mit Diabetes sind die häufigsten Sexualstörungen: Erektile Dysfunktion (ED), Libido-, Ejakulationsund Orgasmusstörungen. Die erektile Dysfunktion ist die dritthäufigste Diabeteskomplikation und kann die Lebensqualität stark beeinträchtigen. „Der Diabetes

$$
\begin{gathered}
\text { „Ein Mann hat Sex, um } \\
\text { den Kopf frei zu } \\
\text { bekommen, eine Frau } \\
\text { braucht einen freien } \\
\text { Kopf für Sex." }
\end{gathered}
$$$$
\text { (Dr. Astrid Tombek) }
$$

in ihren pharmakokinetischen Eigenschaften, sodass für jeden einzelnen $\mathrm{Pa}$ tienten das passende Präparat gefunden werden könne. Bei fehlender Wirkung sollte ein Substanzwechsel erfolgen. Auch kann es sinnvoll sein, den Testosteronspiegel $\mathrm{zu}$ bestimmen, bevor andere Therapiemodalitäten wie SKAT mit erhöht das Risiko für eine erektile Dysfunktion um etwa das Vierfache“, betonte Dr. Frank Merfort, Grevenbroich. Die Prävalenz beträgt in der Gesamtbevölkerung bei 65 -Jährigen $25 \%$, bei gleichaltrigen Diabetikern dagegen über $90 \%$. Es ist deshalb wichtig, so Merfort, das Thema einfühlsam anzusprechen. Eine gute Kommunikationsbrücke sei die Komorbidität - etwa so: „Ihre Begleiterkrankungen lassen vermuten, dass Sie Probleme mit der Erektionsfähigkeit haben.“

Erwartungen des Patienten Im Rahmen dieser Gespräche sollte der Patient über die verschiedenen Therapiemöglichkeiten, die Nebenwirkungen und auch die Kosten informiert weden. „Nach solchen Gesprächen entscheiden sich heute 95\% der Patienten primär für den PDE-5-Inhibitor“, so Merfort. Die vier derzeit verfügbaren Substanzen (Sildenafil, Vardenafil, Tadalafil, Udenafil) unterscheiden sich
Prostaglandin-E1 oder die Vakuumpumpe besprochen werden.

\section{Angst vor dem Versagen führt zur Chronifizierung}

„Die sexuelle Appetenz kann durch das negative Selbstbild als chronisch Kranker gestört werden“, sagte Dipl. Psych. Susan Clever, Hamburg. Gerade bei jungen Typ-1-Diabetikern gelte der Diabetes nicht als „sexy“. Insbesondere bei Frauen können auch aufwendige Vorbereitungen (Abnahmen der Pumpe, Vorbeugung einer Infektion, Empfängnisverhütung etc.) den psychophysiologischen Ablauf bei sexuellen Aktivitäten empfindlich stören. Auch Depression und Stress sind Lustkiller, d.h. sie verstärken die sexuelle Funktionsstörung. „Bei Männern ist oft die Angst vor dem Versagen das eigentliche Problem“, so Clever. Folge sei ein Vermeidungsverhalten, was zur Chronifizierung des Problems beitrage.

Dr. med. Peter Stiefelhagen

- Diabetes Kongress am 26.5.2017, in Hamburg 